\title{
The effect of stimulus strength on the jaw-jerk response in man
}

\author{
G R LEW IS, R P I L C HER, A N D R E M \\ From the Department of Dental Prosthetics, University of Dundee
}

SUMMARY The jaw-jerk response was elicited in seven adult male subjects by tapping on the chin in a downward direction. The magnitude of the applied taps was varied subjectively by the operator, and the downward acceleration of the mandible measured by means of a calibrated piezo-electric accelerometer fixed to the lower anterior teeth. The taps were applied during voluntary clenching by the subject, controlled by means of a force transducer placed between upper and lower teeth. The electrical response of masseter and temporal muscles was found to increase both with increasing stimulus strength and with voluntary clenching force. However, only by averaging a number of responses were clear relationships demonstrated, there being other variables, affecting the muscles independently, which it has not been possible to identify and control. It is concluded therefore, that quantitative analysis of the electromyograms of the jaw closing muscles following chin-tap stimuli is not a satisfactory method for testing for abnormality in excitability of the neuromuscular system involved in the jaw-jerk response.

The jaw-jerk response, elicited in jaw-closing muscles by a downward tap on the chin, is sometimes used as a clinical test of the integrity of the trigeminal neuromuscular pathways. The test is applied in a qualitative fashion, where simply the presence or absence of a muscle response is recorded, using electromyographic techniques.

Some attempts to quantify the response of the masseter muscle have been made. Many of these have been concerned with the latency. ${ }^{1}$ Difficulties of measurement arise, both in assessment of the moment of application of the stimulus, and of detection of the first evidence of a response. The response itself is followed by a silent period when the muscle is active at the time of stimulation, as in other muscles. ${ }^{2}$ The onset and duration of this silent period have also been investigated for the masseter muscle. ${ }^{1}$ Furthermore the silent period duration in jaw-closing muscles has been examined in patients with muscular disorders of the region (tcmporomandibular dysfunction).$^{3-5}$ The results are conflicting, and in any case neither the origin of the silent period, nor the relationship of its

\footnotetext{
Address for reprint requests: Dr $\mathbf{R}$ Yemm, Department of Dental Prosthetics, Dental School, University of Dundee, Dundee DD1 4HN, Scotland.

Accepted 31 January 1980
}

duration to preceding excitation of the muscle are clear, in these or other muscles. ${ }^{6}$

Quantitative studies of the jaw muscle excitation following the chin-tap stimulus are incomplete. Goodwill ${ }^{7}$ measured the amplitude of the electrical response of the masseter muscle, but controlled neither the stimulus magnitude nor the degree of voluntary contraction of the muscles at the moment of stimulation. Wide variations in the response were reported. More recently, Hannam, ${ }^{8}$ using an elegant system to control the voluntary contraction of the jaw-closing muscles at the moment of stimulation, and to administer a constant stimulus, showed, on average, a close relationship between amplitude of masseter muscle response and voluntary activation. These experiments did not include investigation of the effect of changing the simulus strength; also, the method of initiation of the stimulus at a preselected point during an increasing voluntary clench precluded examination of the response when the subjects were relaxed.

Quantitative analysis of the jaw-jerk response in a study of multiple sclerosis patients has been attempted. ${ }^{9}$ Latency, amplitude and duration of the response were recorded. Evidence is reported of an increase in latency, or absence of response, 
sometimes only on one side. Amplitude and duration however, were not found to be useful measurements, but, as in a related study of normal subjects, ${ }^{7}$ neither the stimulus strength nor the level of activity of the muscle at the moment of stimulation, were controlled.

The present experiments were designed to investigate the possibility of more quantitative use of the jaw-jerk response, by measurement of the effects of stimulus strength upon the jaw-jerk response over a wide range of voluntary jaw-closing activity. In addition, recordings were made simultaneously from masseter and temporal muscles, to enable a comparison of the responses of the two muscles. In previous reports little or no attention has been paid to the response of the latter muscle.

\section{Methods}

\section{Measurement of stimulus}

The stimulus was measured directly, as acceleration, by means of a miniature piezo-electric accelerometer attached to the lower jaw. The device was rigidly located behind the lower incisor teeth, in the mid-line, by means of an acrylic resin appliance clasped to molar and pre-molar teeth. The accelerometer, which was mounted so as to be sensitive to acceleration in opening and closing directions, provided a more objective measure of the stimulus strength than hitherto, where the most common method has been to quantify the magnitude of the tap applied to the chin. ${ }^{6}$

Taps were applied to the point of the chin via the operator's finger, ${ }^{1}$ using a reflex hammer incorporating a micro-switch to provide a trigger pulse. The magnitude of the stimulus was measured as the peak downward acceleration of the mandible (fig 1). The accelerometer and its amplifier were calibrated against a standard, and responded in a linear fashion over the range of acceleration and frequency involved in the present experiments.

\section{Control of voluntary muscle activation}

A calibrated strain gauge transducer was placed between the upper and lower right canine and first pre-molar teeth, using dental impression compound on it's upper beam to ensure reproducible positioning. The signal from the transducer amplifier was displayed on a large centre zero meter, placed in front of the subject. The transducer amplifier offset was adjusted so that the meter zero corresponded to desired force levels, and the subje:t asked to maintain the meter pointer at

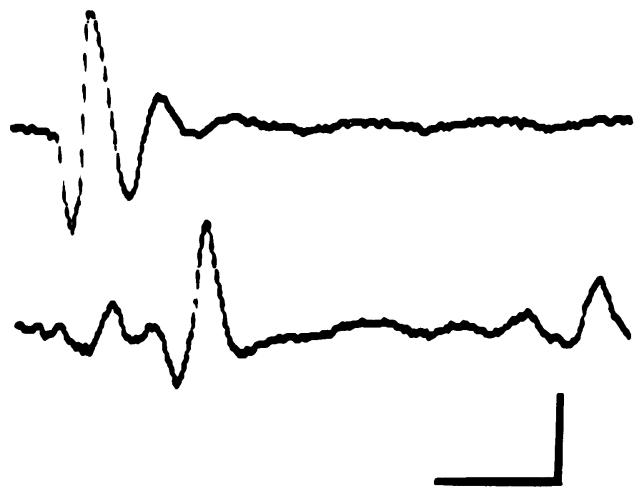

Fig 1 Accelerometer signal (upper trace) and temporal muscle EMG following a tap on the chin while the subject was exerting the equivalent of $2 \mathrm{~kg}$ on the force transducer. The amplitude of the first downward deflection of the accelerometer signal was measured. Calibrations: Horizontal: $8 \mathrm{~ms}$, Vertical: $50 \mathrm{~m} / \mathrm{s}^{2}$ (accelerometer) $0.15 \mathrm{mV}(E M G)$

zero by clenching on the transducer. Positive and negative full scale deflections of the meter corresponded to $\pm 250 \mathrm{~g}$. The dimensions of the transducer determined the jaw position, which was approximately $8 \mathrm{~mm}$ open from the position of maximum closure.

\section{Measurement of muscle response}

The response was measured using electromyographic techniques (EMG). Two silver disc electrodes $(9 \mathrm{~mm}$ diameter) were fixed to the skin overlying the right masseter muscle, over the centre of the muscle, $2 \mathrm{~cm}$ apart in the general direction of the muscle fibres. In most experiments two further electrodes were applied to the right temporal muscle, also $2 \mathrm{~cm}$ apart, over the middle fibres.

The signal from the electrodes was amplified (differential, frequency range $10-1000 \mathrm{~Hz}$ ), and tape recorded, together with the trigger pulse from the stimulation hammer, the accelerometer signal, and in some experiments the force transducer signal.

The magnitude of the individual responses was obtained from the recording by integration of the EMG, over the time period shown by preliminary inspection to include the whole of the wave form of the response. This was commonly of 10 to $12 \mathrm{~ms}$ duration, starting $5 \mathrm{~ms}$ after the trigger pulse. At the end of each integration period the integral was stored in a sample/hold device and subsequently prin ed by a recording digital voltmeter. The integrator reading in the absence of a re- 
sponse was obtained by triggering the integration and recording sequence at times when no stimuli were being applied; this operation was performed at each force level demanded of the subject. The voltage by which an integral exceeded this reference level was thus the indication of magnitude of the response.

\section{Experimental procedure}

The subjects were seven adult males, age range 18 to $45 \mathrm{yr}$, with good natural dentitions, and free of local muscle or joint dysfunction. The experiments were carried out with the subjects seated upright in a dental chair with a headrest. Each subject was asked to maintain forces corresponding to loads of $\frac{1}{2}, 1,2$ and $3 \mathrm{~kg}$ upon the transducer. The sequence in which these were demanded was randomised to obviate the chances of systematic effects of fatigue. In addition, for one period the subject was asked to relax with the transducer remaining in place between the teeth. During this time the meter was removed from the subject's view. The subject was judged to be relaxed by observation of minimal activity on the EMG signals.

The procedure at each force level was first to allow the subject a brief practice at maintaining the target force, and then to apply stimuli in groups of five at approximately three intervals, with a rest period between each group. The stimulus magnitude was randomised subjectively by the operator, during a total of 30 chin-tap (six groups of five). The three sec interval between stimuli was found sufficient to allow the subject to reestablish a steady clenching force.

\section{Results}

At each voluntary clenching force, a tendency was found for the individual responses (fig 1) to increase with increasing stimulus strength. Results from the masseter muscle for one subject at two force levels, are shown graphically in fig 2 . This example shows a large degree of scatter of the data, a finding which was characteristic of all subjects, and for both muscles tested. The availability of corresponding results from masseter and temporal muscles enabled a comparison of the two series to determine where there was a relationship. Fig 3 shows the response integrals of the temporal muscle plotted against those from the masseter, for all data obtained from one subject at force levels of $\frac{1}{2}$ and $3 \mathrm{~kg}$. Although a relationship is just apparent, there was independent variation in the response of the two muscles.

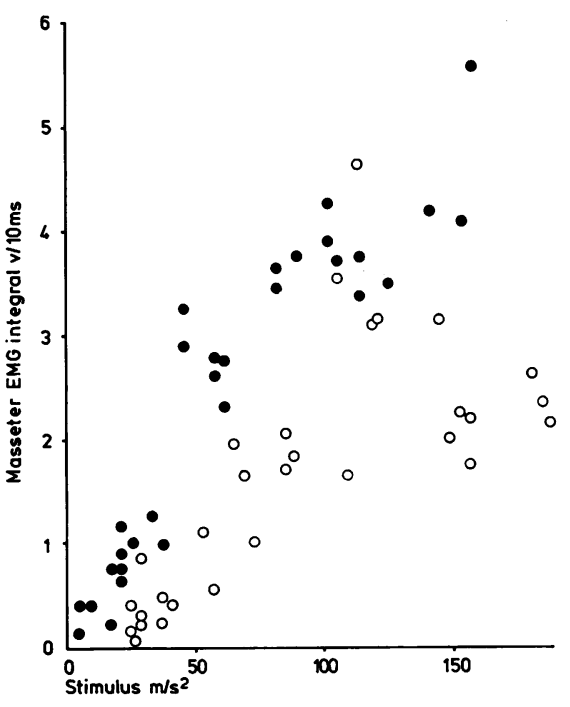

Fig 2 The response of the masseter muscle to stimuli of varying strength. Data plotted as open circles were obtained while the subject exerted $\frac{1}{2} \mathrm{~kg}$ on the transducer. Filled circles are for data obtained with background activity equivalent to $3 \mathrm{~kg}$. The scale of $E M G$ response is the output of the integrator, no attempt having been made to relate this to the voltage recorded at the electrodes.

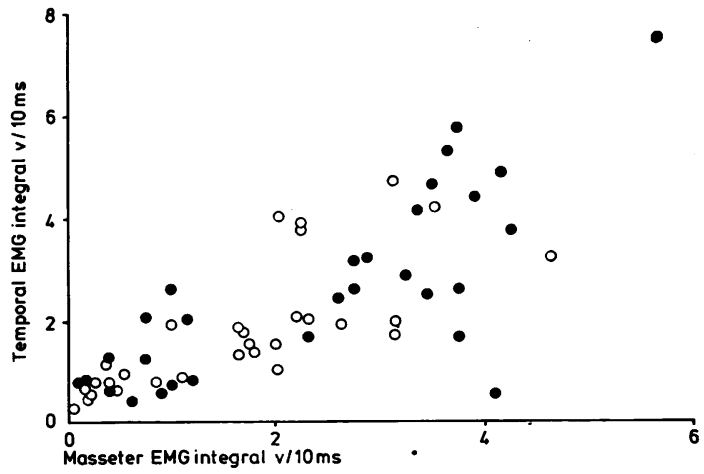

Fig 3 The responses of masseter and temporal muscles, obtained with background activity equivalent to $\frac{1}{2} \mathrm{~kg}$ (open circles) and $3 \mathrm{~kg}$, with a wide range of stimulus magnitude. The data is from the same subject as fig 2.

Responses to stimulation during relaxation were also variable. Particularly at lower levels of stimulation, there was often no detectable response. With larger stimuli, responses were more consistently visible and measurable. Fig $4 \mathrm{~A}$ shows the acceleration signal and the temporal muscle 

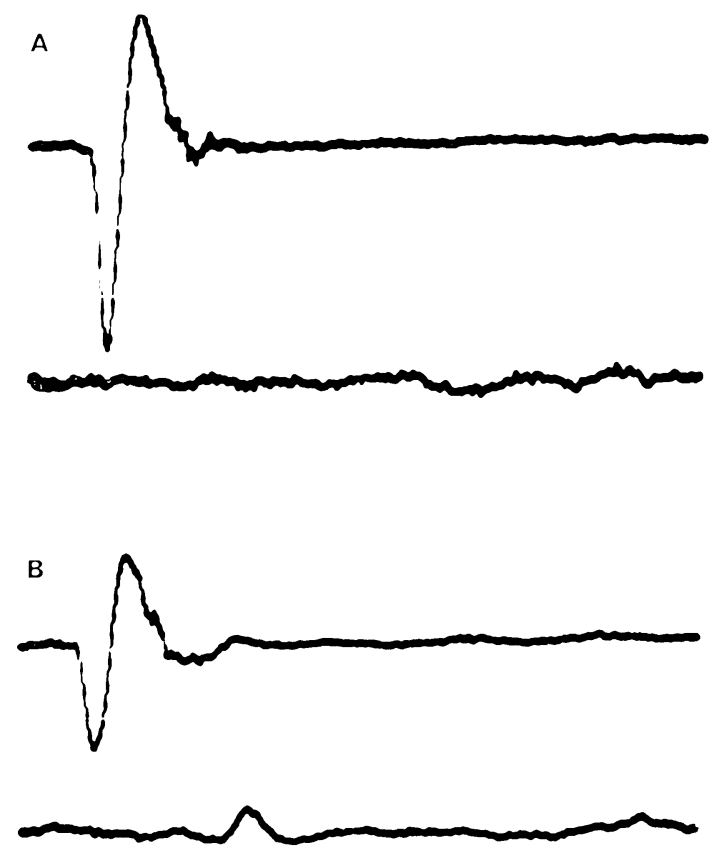

Fig 4 The two upper records $(A)$ show that after a large tap to the chin producing a large downward acceleration, there was no detectable temporal EMG response during relaxation. In contrast during maintenance of $a \frac{1}{2} \mathrm{~kg}$ clench the example in two lower records $(B)$ shows a small but clear response to a tap causing approximately half the downward acceleration of the lower jaw. Calibrations:

Horizontal $8 \mathrm{~ms}$, Vertical Accelerometer $(A$ and $B)$ $50 \mathrm{~m} / \mathrm{s}^{2}, E M G(A) 0.075 \mathrm{mV}$, (B) $0.15 \mathrm{mV}$.
EMG following a large tap to the chin while relaxed, with no evidence of muscle response. For comparison, Fig 4B shows corresponding traces from the same subject following a smaller chintap, while exerting a force equivalent to $\frac{1}{2} \mathrm{~kg}$ on the transducer when a small but clear response occurred. One subject in particular (not the subject of fig 4) failed to respond in either muscle to stimulation when relaxed although his results during voluntary clenching were comparable to those of the remaining subjects.

\section{Discussion}

The results of this study show that the magnitude of the response of the masseter and temporal muscles following a downward tap on the chin depends in part upon the peak acceleration of the mandible in an opening direction. As such, the results are complementary to those showing that the response is dependent upon the level of voluntary clench at the time of stimulation, ${ }^{\varsigma}$ where a close relationship was demonstrated between clenching force and response, when a number of responses were averaged. The results of the present experiments do not lend themselves readily to averaging, because the stimulus strength was varied at random over a wide range. As an approximation, however, the stimuli can be grouped over a number of narrow ranges for this purpose. When this was done, a clearer picture of the tendency for the responses to vary with both stimulus strength and force of voluntary clench emerges (fig 5). Here, for one subject, the average masseter response to the smallest stimuli applied is shown to increase with clenching force. Larger stimuli produced higher average responses, similarly related to the level of voluntary muscle

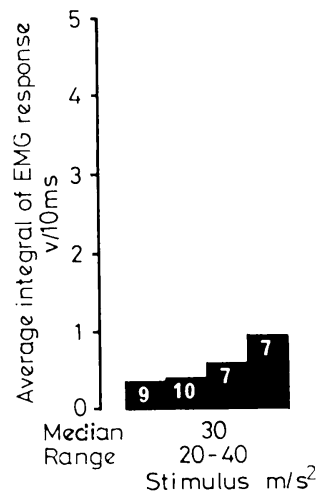

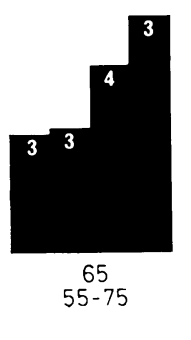
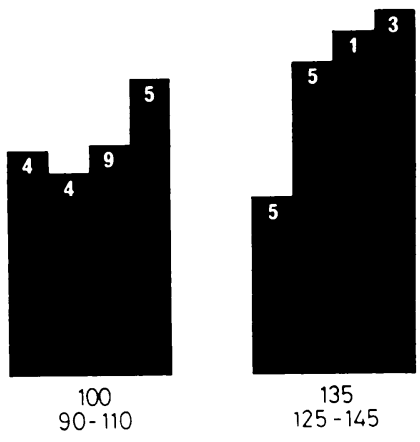

Fig 5 Average data for one subject. The four block diagrams each correspond to a range of stimulus strength (the median and range is presented on the horizontal axis). Each block consists of the average masseter muscle response at ${ }_{2}^{1}$. 1, 2 and $3 \mathrm{~kg}$ clenches. The number of responses in each block varies, owing to the experimental procedure, and is given on diagram. 
activation. It must be emphasised that this procedure masks the variability of individual responses.

The origin of the variation in magnitude of the response is not clear. A number of possibilities have been considered. Control of the voluntary clench was not precise, since subjects were unable to maintain a perfect correspondence of the meter needle and the centre zero, so that some stimuli were applied at force levels above or below the target, or at times when the force was rising or falling. Although this may be significant, it cannot account for all the data scatter observed, in view of the apparently independent variation of masseter and temporal muscle data. A similar deduction may be made from the variability observed by Hannam, ${ }^{8}$ where the force level was presumably more accurately controlled and where the voluntary force level was always rising, although possibly at different rates, at the moment of stimulation.

It seems more likely that the response variability is related to normal processes within the responding muscles. The response is probably due to synchronisation, by way of muscle spindle afferents, of motor units active at the time of the chin-tap, and simultaneous stimulation of those not actually discharging but whose threshold is low (that is, units which would be recruited to achieve a small increase in voluntary contraction). Clearly the excitability of motor units in both these categories must vary from moment to moment. A further possibility is that the proportion of the total voluntary force produced by the different jaw-closing muscles is varying during the clench, resulting in changes in relative numbers of units excitable in the different muscles involved in the clench.

An alternative to measurement of the EMG response of individual muscles would be to record the response of the jaw-closing muscles as a group, by reference to the force transducer signal. This was recorded in the last two experiments in the present series, and showed both the immediate result of the applied tap, and a subsequent increase in the force (fig 6). The latter must be due to the reflex muscle response because it occurred much more quickly than the reaction time, for this muscle, of $120 \mathrm{~ms}$ or more. ${ }^{10}$ Further experiments of this type are planned, and these may produce a more reliable, and therefore more useful, measure of the jaw-jerk response.

The frequent absence of a response when the subjects were relaxed is consistent with finding for other muscles. ${ }^{11}$ At least under the conditions of the present experiments, the threshold of motor

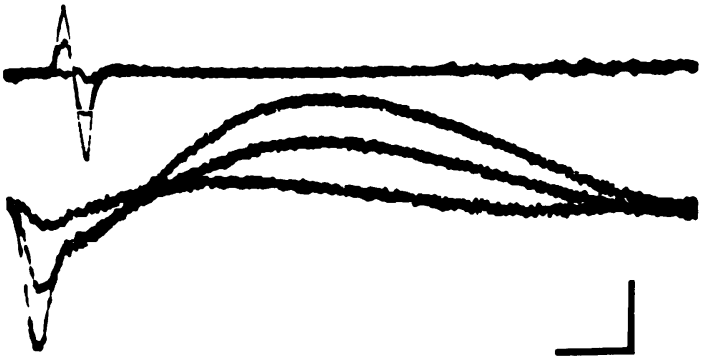

Fig 6 Three superimposed records of the masseter muscle EMG (upper trace) and the force transducer signal. The force signal shows an early downward deflection, indicating a reduced force level, corresponding to the chin-tap. Subsequently the force level can be seen to have risen. Calibrations: Horizontal $10 \mathrm{~ms}$, Vertical EMG $1.25 \mathrm{mV}$, Force $6.25 \mathrm{~g}$.

units of the masseter and temporal muscles must have been such that no motor units, or only an undetectably small number, were excitable. The chin-tap stimulus tends to synchronise the discharge of active units of the muscles. Therefore, under the relaxation conditions of the present experiment, it is reasonable to conclude that the muscles tested were sometimes inactive, since even small units recruited to develop low forces are sometimes visible individually on the surface EMG, ${ }^{12}$ and synchronisation of a number of such units would be expected to increase their detectability still further above amplifier noise levels.

We thank Mr DD Chalmers for preparation of the illustrations.

\section{References}

1 Widmalm SE, Hedegard B. Reflex activity in the masseter muscle of young individuals. I. Experiment procedure-results. J Oral Rehabil 1976; 3: 41-55.

2 Hammond PH, Merton PA, Sutton GG. Nervous gradation of muscular contraction. Br Med Bull 1956; 12:214-8.

3 Bassette R, Bishop B, Mohl N. Duration of masseteric silent period in patients with TMJ syndrome. J appl Physiol 1971; 30:864-9.

4 Griffin CJ, Munro RR. Electromyography of the masseter and anterior temporalis muscles in patients with temporomandibular dysfunctions. Arch Oral Biol 1971; 16:929-49.

5 Widmalm SE. The silent period in the masseter muscle of patients with TMJ dysfunction. Acta Odont Scand 1976; 34:43-52. 
6 Matthews B. Mastication. In: Lavelle CLB ed. Applied physiology of the mouth. Bristol: J Wright, 1975; 199-242.

7 Goodwill CJ. The normal jaw reflex: measurement of the action potential in the masseter muscles. Ann Phys Med 1968; 9:183-8.

8 Hannam AG. Effect of voluntary contraction of the masseter and other muscles upon the masseteric reflex in man. J Neurol Neurosurg Psychiat 1972; 35:66-71.

9 Goodwill CJ, O'Tuama L. Electromyographic recordings of the jaw reflex in multiple sclerosis.
J Neurol Neurosurg Psychiat 1969; 32:6-10.

10 Yemm R. Reflex jaw opening following electrical stimulation of oral mucous membrane in man. Arch Oral Biol 1972; 17:513-23.

11 Marsden CD, Merton PA, Morton HB. Servo action in the human thumb. J Physiol (Lond). 1976; 257:1-44.

12 Yemm R. The representation of motor-unit action-potentials on the skin-surface electromyograms of the masseter and temporal muscles in man. Arch Oral Biol 1977; 22:201-5. 